\title{
SELECTION PROCESS OF PROTECTION MEASURES FOR LAYERED DEFENSE SCHEME AGAINST EMP THREATS
}

\author{
Sang-Pil Cheon ${ }^{1}$, Keun-Seog Park ${ }^{2}$, Yu-Hyun Kim ${ }^{3}$ and Jung-Ho Eom ${ }^{4}$ \\ ${ }^{1}$ Dept. of Military Studies, Daejeon University, Daejeon, Republic of Korea \\ ${ }^{1}$ skyfeel69202@naver.com, ${ }^{2}$ pksm06@naver.com, ${ }^{4}$ eomhun@gmail.com
}

\begin{abstract}
Recently, EMP (Electromagnetic Pulse) bombs, a core weapon system for electronic warfare, have been highlighted. EMP bombs use high-power electromagnetic waves that are intentionally generated by electromagnetic wave generators to stop the operation of the target information communication system or to paralyze its function. In this paper, we propose a process to determine the most appropriate protection measures for the layered defense scheme. This process determines the scope of the system to be protected, performs a risk assessment for the system, identifies the level of protection, and selects a protection measure that can reduce the risk to the protection level. In addition, it divides the protection measures by the defense zone, performs the cost effectiveness analysis, and decides the appropriate protection measures considering the intention of the commander and the utilization and contribution of the system.
\end{abstract}

Keywords - EMP; Electronic Warfare; Radiative/Conductive Threats, Layered Defense, Selection Process

\section{INTRODUCTION}

In 2017, a CIA expert told in the Congressional National Security Subcommittee, "If North Korea attacks the United States with a nuclear EMP, it could kill $90 \%$ of Americans because of starvation, disease, and social collapse." The Korean Defense Minister also said in the National Assembly that "the main command forces of the military, including the Joint Chiefs of Staff, are equipped with electromagnetic shielding capabilities, but defense facilities are not perfect for battalion or division." [1,2]. The damage effect of EMP attack may cause paralysis or malfunction of power distribution system, telecommunication network, various electronic equipment, electronic devices, etc., to the ground radius up to about $600 \mathrm{~km}$. In addition, services such as telecommunication and public power supplies may be temporarily suspended or be occurred long-term outages. In 1962, when a nuclear warhead exploded 400 kilometers above Johnston Island in the Pacific Ocean, 300 street lights were shut down and communications stopped in Hawaii, 1,400 kilometers away. In addition, malfunctions occurred in electronic equipment and the low-orbit satellites of the US military were shut down $[3,4]$

The types of EMP are nuclear EMP and non-nuclear EMP, and EMP threats are classified into radiative and conductive threats. Therefore, it is necessary to establish a protection policy and countermeasures for the military information and communication system according to the kinds and threats of EMP. The ROK military's information and

Received: January 12, 2019

Reviewed: March 4, 2019

Accepted: March 6, 2019

* Corresponding Author 
communication system is a system directly linked to military operations, such as battlefield control systems, combat command control systems, and intelligence transmission systems, and cannot guarantee defense security if enemy attacks with EMP bombs. In spite of this, the current EMP protection measures are facility-oriented protection measures, and there are insufficient protective equipment and technology oriented protection measures considering the characteristics of the unit system and information communication system. The protection equipment and technology need to be developed specifically for the military information communication system against the elaborate EMP threats. In the private sector and military, there were constructed a shielding technology for protection against radiative threats and an overcurrent protection technology using HEMP (High-altitude Electromagnetic Pulse) filters for protecting against conductive threats. In modern warfare, technology (equipment) oriented protection measures should be established rather than costly and inconvenient mobility protection facilities because C4I is installed to small units. The C4I (Command, Control, Communication, Computer, and Intelligence) is the most important command control system in the battlefield, and is the target of jamming, hacking and physical attacks. Since the military information and communication system changes position by movement of units, it is necessary to establish EMP protection measures based on protection technologies and equipment such as electromagnetic shielding technology and conductive pulse protection circuit technology rather than facility-oriented protection measures. Therefore, it is possible to satisfy the appropriate level of protection and also cost effective [4-7]

We propose a process to determine the appropriate protection measures for the layered defense scheme. The layered defense scheme is prevented by protection facilities in defense zone 1 and 2 . In defense zone 3, protection measure is to minimize the damage by using protection technology and equipment, respectively. This paper describes the definition and characteristics of EMP in Chapter 2 and describes the EMP protection measures in Chapter 3. In Section 4, we propose a selection process for the layered defense, and conclude in Section 5.

\section{THE DEFINITION AND CHARACTERISTICS OF EMP}

The EMP (Electromagnetic Pulse) is conceptualized by the electromagnetic wave source. EMP is classified into nuclear high-power electromagnetic wave by nuclear explosion and non-nuclear high-power electromagnetic wave which generates electromagnetic wave directly by electronic bomb or high-power electromagnetic wave generator. EMP by nuclear bombs means strong electromagnetic waves generated by ionization of atoms in the atmosphere by gamma rays generated when a nuclear weapon explodes. EMP by EMP weapon means a high output electromagnetic wave intentionally generated by an electromagnetic wave generator such as an EMP weapon. EMP by natural phenomena is caused by electromagnetic waves such as lightning, natural phenomenon, near-static discharge, and solar magnetic storm. In addition, electromagnetic interference (EMI) is a phenomenon that affects the functions of other electronic devices, such as interfering with images and causing malfunctions of computers and electronic devices. In this paper, we limit non-nuclear electromagnetic wave, especially EMP weapon for intentional attack. The non-nuclear EMP is an electromagnetic wave that generates intentional and malicious radiative electromagnetic waves for the purpose of war, terrorism, etc., thereby damaging or malfunctioning electronic equipment, etc. It is called high power electromagnetic (HPEM) or intentional electromagnetic interference (IEMI) [4,6,8,9]. The following table shows the characteristics of an EMI, EMP by nuclear explosion, and intentional EMP. 
Table I. Comparison of electromagnetic pulse characteristics [9]

\begin{tabular}{c|c|c|c}
\hline Type & EMI & HEMP & HPEM \\
\hline $\begin{array}{c}\text { Frequency } \\
\text { bandwidth }\end{array}$ & $\begin{array}{c}30 \mathrm{MHz}-6 \mathrm{GHz}(\mathrm{RE}) \\
9 \mathrm{kHz}-30 \mathrm{MHz}(\mathrm{CE})\end{array}$ & $\sim 500 \mathrm{MHz}$ & $100 \mathrm{MHz}-10 \mathrm{GHz}$ \\
\hline $\begin{array}{c}\text { intensity of } \\
\text { electromagnetic }\end{array}$ & $3 \mathrm{~V} / \mathrm{m}$ & $50 \mathrm{kV} / \mathrm{m}$ & $\begin{array}{c}\text { Hundreds of V/m- } \\
\text { Hundreds of } \mathrm{kV} / \mathrm{m}\end{array}$ \\
\hline Impact distance & $\sim$ Tens of $\mathrm{m}$ & $\sim$ Hundreds of km & \begin{tabular}{c}
$\sim$ Hundreds of $\mathrm{m}$ \\
\hline Impact Objects
\end{tabular} \\
$\begin{array}{c}\text { Electrical and } \\
\text { electronic equipment }\end{array}$ & $\begin{array}{c}\text { Power / communication } \\
\text { network }\end{array}$ & $\begin{array}{c}\text { Major buildings such as IDC, } \\
\text { nuclear power plants }\end{array}$ \\
\hline Source & $\begin{array}{c}\text { Electrical and } \\
\text { electronic equipment }\end{array}$ & $\begin{array}{c}\text { Nuclear explosion } \\
\text { E-bomb, etc. High power } \\
\text { radiator }\end{array}$ \\
\hline
\end{tabular}

Modern society will be super connected, cross domain, and super intelligent society that electronic equipment parts are mostly miniaturized and integrated, all devices are connected as a single network, and the control system of national infrastructure such as communication network and power network composed of computer and various applications. In addition, military and national security related systems will be exposed to EMP threats by linking with C4I, and when an EMP attack occurs, there will be enormous damage and confusion throughout the national infrastructure as well as national security. EMP is regarded as a major weapon system in modern warfare, which is an asymmetric power that emits powerful electromagnetic waves in a short period of time and paralyzes all electronic devices in a short time to disable command, control and communication systems. The effect can paralyze and damage the core chips of all electronic devices such as aircraft, combat vehicles, ships, radios, mobile devices, computers, and watches, as well as electricity cuts [8]

As ICT technology develops rapidly, social reliance on mission-critical or safety critical electrical and electronic systems in national infrastructures increases, but vulnerabilities to electromagnetic wave threats increase and risks become more severe. In addition, as the level of electromagnetic wave susceptibility of IT systems operating at high frequency and low voltage is lowered to improve the efficiency of electronic equipment and information systems, vulnerabilities due to intentional electromagnetic interference are also increasing. As the high-power electromagnetic wave related technologies such as high energy RF generator and high efficiency antenna are developed, the spread of HPEM generation sources is rapidly spreading and the functions are also diversified. It is expected that electronic attacks using HPEM will be increased because easy development of high power electromagnetic wave generation equipment, exposure to electromagnetic waves, and the ability to stealthy attack outside the physical boundary due to the characteristics of electromagnetic waves. Intentional attacks by EMP can cause various data and information to be destroyed in a moment, and the damage area and extent are also very wide [8,9]. In the military, a variety of high-power electromagnetic wave generators are being developed for electronic attacks that do not cause human casualties and can reduce attack costs. EMP is being developed in accordance with the target of attack and the means of transportation. The following table shows high-power electromagnetic wave generators in accordance with the means of movement. 
Table II. High-power electromagnetic wave generators by the means of movement [9]

\begin{tabular}{c|c|c|c|c}
\hline Type & Portable & \multicolumn{2}{|c|}{ Vehicle } & Aircraft \\
\hline Generator & $\begin{array}{c}\text { It can be used at close } \\
\text { range, but the field strength } \\
\text { is weak and the threat level } \\
\text { is low. }\end{array}$ & $\begin{array}{r}\text { Moving and supplying power by } \\
\text { using the vehicle. The size of the } \\
\text { EMP device varies depending on the } \\
\text { size of the vehicle. }\end{array}$ & $\begin{array}{c}\text { It is moved by airplane } \\
\text { and dropped into the } \\
\text { air above the target. }\end{array}$ \\
\hline $\begin{array}{c}\text { Electronic intensity } \\
\text { (at } 100 \mathrm{~m})\end{array}$ & $1.2 \mathrm{kV} / \mathrm{m}$ & $3.3 \mathrm{kV} / \mathrm{m}$ & $500 \mathrm{kV} / \mathrm{m}$ & Hundreds $\mathrm{kV} / \mathrm{m} \sim$ \\
\hline
\end{tabular}

Recently, with the development of high-power electromagnetic wave generator technology, electromagnetic wave intensity is very powerful. As high-power sources of electromagnetic waves become more diverse, the attack success rate is increased by finding a method that can effectively infiltrate the target system. The infiltration method is a way of passing through air and a way of infiltrating through a pipe or a cable. In other words, it is distinguished radiative threats and conductive threats. Radiative threats generate high-power electromagnetic waves in the air and penetrate through structurally open paths such as doors, windows, vents, and pipes of facilities where electronic equipment is located. Radiative electromagnetic waves cause performance degradation or functional paralysis of all electronic equipment and control systems within the threat radius due to electromagnetic pulse radiation. Conductive threats generate strong currents due to the generation of high-power electromagnetic waves and infiltrate through electrically infiltrating paths such as power lines, telecommunication lines, and antennas that enter the target facility. It causes to stop operation or degrade performance of various electronic devices and control system connected to transmission lines and communication lines [4-6,11]. The following figure shows the EMP infiltration method. Mobile communication devices are more vulnerable to radiative threats than conductive threats because they do not have communication or power lines to provide power.

As mentioned earlier, there are two main ways for HPEM to reach the electronic devices that control the operation of battlefield management system. One is by the direct illumination of the devices and the other is by the coupling of the HPEM fields to cables and connection lines, producing conducted transients that can go over the susceptibility of the connected electronic devices. A current injection of an HPEM directly is also a threat to command and control center in buildings if access to the entrance zone for power and communications cables is not restricted [12].

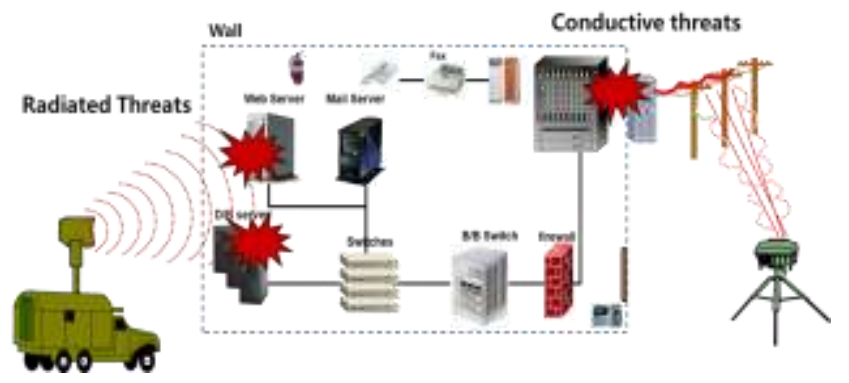

Fig. 1 Types of EMP infiltration. 


\section{PROTECTION MEASURES AGAINST EMP THREAT $[3,4,10,13]$}

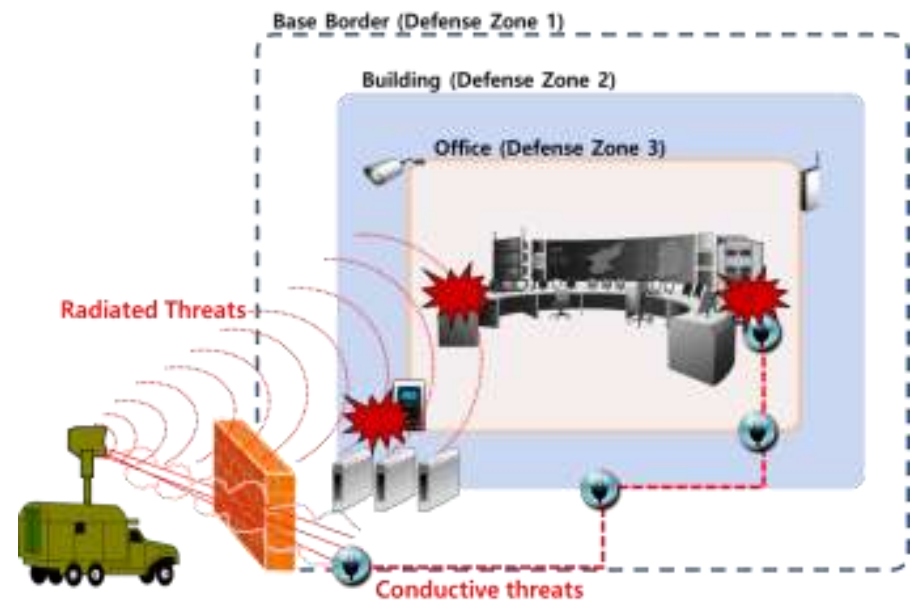

Fig. 2 Defense Zone against EMP Threat

EMP protection for major facilities in South Korea are applied simultaneously with protection measures against radiative threats and conductive threats, and are built protection facilities for military command control units and government communication facilities. The protection measures apply both methods together. Based on the basic concept of protection technology, first protection measure is to construct a protection facility that can guarantee radiative threat protection performance, the other measure is to install protection equipment to guarantee the protection performance against the conductive threats to various power and communication cables entering the inside of the protection facility.

Methods to prevent radiative threats include the establishment of electromagnetic shielding facilities, the installation of the important battlefield management system in an underground facility, and the securing of sufficient distance from the outside. Electromagnetic wave shielding is a way of blocking or attenuating the inflow of electromagnetic wave as sealing a space requiring protection by a conductive material. The shielding facilities with special structure are installed in entrance, ventilating opening and penetration hole for entrance of optical cable. If the important server and command \& control system are installed in the underground space, it can provide the natural shielding effect by the geographic feature and the rock, and it can attenuate the intensity even though the high power electromagnetic wave penetrates it. Securing the separation distance is a way of attenuating the intensity of the high power electromagnetic wave by securing a sufficient distance from the outside because the intensity of the electromagnetic wave is inversely proportional to the square of the distance.

A method to prevent a conductive threat is to prevent an overcurrent generated by a high-power electromagnetic wave from flowing into an internal system through an external power line, a communication line, and so on. The cut-off filter technology is a way of blocking the overcurrent generated by the high-power electromagnetic wave as installing a filter equipped with an overcurrent protection device in various power and signal / control lines that are drawn into the inside of the protection facility. The overcurrent protection device used for the filter is MOV (Metal Oxide Varistor) or GDT (Gas Discharge Tube). They normally have impedance characteristics of several $M \Omega$ to several hundreds of $\mathrm{M} \Omega$ and do not affect the data communication of various power supplies and signal / control lines, but when a conductive threat situation occurs, the impedance is changed from tens of $\mathrm{m} \Omega$ to several $\mathrm{m} \Omega$, and thus it can block the overcurrent caused by the conductive threat. The most important technology in the signal 
and control filter is to cut off the high-power electromagnetic wave signal and transmit the used frequency signal necessary for communication without distortion. A method of changing an electric signal to an optical signal is to block intrusion of a high-power electromagnetic wave signal and leakage of electromagnetic wave using a photoelectric conversion module. A photoelectric conversion module is installed at the point of entry of the main line and the point connected to the system. It is a method of enhancing susceptibility by adding an anti-intrusion module to system components to prevent malfunction caused by the injection of conductive electromagnetic waves into the system.

\section{PROPOSED DECISION-MAKING PROCESS FOR LAYERED DEFENSE SCHEME}

\subsection{LAYERED DEFENSE SCHEME AGAINST EMP THREATS [16]}

It needs to establish protection measures from an outside to inside to protect battlefield management system because most of the EMP threats originate from the outside. An important battlefield management system is located in the safest space in a building or in an underground facility. As shown in the following figure, it is only necessary to establish protection measures by dividing into defense zones.

In the first defense zone, there is installed the protection fence or external security facility. Protection fence to prevent radiative threats shall be made of equipotential structures by assembling or welding shielded structures made of conductive materials such as steel. Also, by connecting to the ground at the point where the shielding facility is installed, high-power electromagnetic pulses generated from the outside can be blocked. And it should be constructed as a structure that discharges the electromagnetic waves flowing to the surface of the shielding fence to the ground. This defensive zone is primarily aimed at defending against radioactive threats and protection fences are not effective against conductive threats.

In the second defense zone, there are installed protection structures and equipment in the building. The protection equipment utilizes a shielding rack, a shielding tent, and a shielding enclosure to protect the server, the power supply, the communication network, and the equipment. In this defense zone, the remaining radioactive threats are minimized and the conductive threats are primarily prevented.

In the last defense zone, there are protection equipment and technology based on the office and the system. Protection measures should be established against conductive threats rather than radiative threats in the last zone. Protection technologies utilize overcurrent protection devices used in HEMP filters to prevent conductive threats. Since mobile communication devices are not fixed in position, it is necessary to attach a protection device or to apply technology to protect the device.

\subsection{SELECTION PROCESS TO DECIDE THE OPTIMAL PROTECTION MEASURE}

For effective protection against EMP threats, there are considerations before choosing a protection measure. Protection measures that reduce the intensity of electromagnetic waves to the lowest level cannot be the best protection measure. For example, it is possible to reach an appropriate level of protection when installing moderate protection equipment in a defense zone rather than establishing expensive protection facilities. The following table shows the proposed the section process to decide the optimal protection measure. 


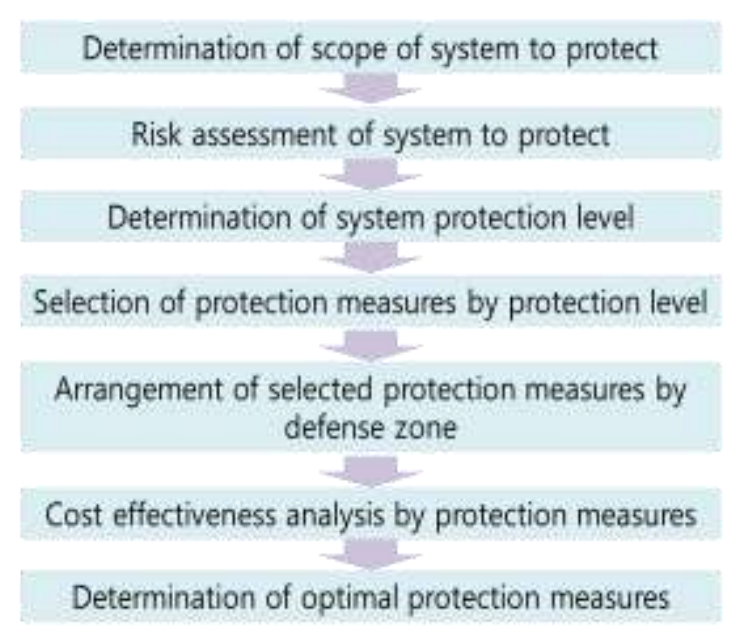

Fig. 3 Selection Process to decide the optimal protection measure

(1) Determination of scope of system to protect: It restricts to critical information and communication system assets that could lead to disruption in battlefield management and military operations resulting in delays, failures, and failures

(2) Risk assessment of system to protect: This step consists of vulnerability and threat analysis, and risk assessment. It is analysed the newly derived vulnerability by combination of various vulnerabilities or inherent vulnerabilities of system vulnerabilities based on the result of comparing the threat level of high power electromagnetic waves with the electromagnetic wave susceptibility of the system or based on the result of the electromagnetic compatibility test. Threat analysis derives and classifies threats based on high-power electromagnetic pulse generation technology, high power antenna technology, electromagnetic wave generator function, and vulnerability level of system. The risk assessment calculates and prioritizes the risk level for each system considering the results of vulnerability and threat analysis, level of contribution to defense and military operations, and utilization

(3) Determination of system protection level: The appropriate level of protection is determined by referring to the scale and characteristics of the system, the level of protection by the type of system, and the domestic / international standards related to the protection measures against the EMP threats

(4) Selection of protection measures by protection level: they are selected protection measures such as protection facilities, equipment, and technologies that meet the level of protection per system and can reduce risks.

(5) Arrangement of selected protection measures by defense zone: The protection measures are classified according to the layered defense scheme as described above. Because the layered defense scheme has been established from the outside to the inside, the first defense zone should be built based on the facility and the last defense zone and system should be installed based on equipment and technology.

(6) Cost effectiveness analysis: The cost effectiveness analysis is carried out based on the economic cost such as the installation cost, the operating cost, and the risk reduction effect among the selected protection measures.

(7) Determination of optimal protection measures: The most appropriate protection measure should be selected for each defense zone. However, protection measures cannot be selected only by risk assessment, cost-effectiveness analysis, and risk reduction rate. 
The final decision must be decided by considering the contributions of military operations, relevance to other systems, strategic and operational significance, and commander intentions.

\section{CONCLUSION}

EMP threats occur over a wide band from low frequencies to high frequencies. As a result, the circuits in various communication systems are destroyed and the bonding state in the semiconductor elements is damaged. And, when exposed to the outside by an antenna and a cable, a high-power interference signal is generated, resulting in a malfunction in the overall signal flow.

High power electromagnetic waves are momentary electromagnetic shock waves with strong energy and can cause malfunction or physical destruction of electronic devices. Critical infrastructures and facilities, including railways and airports, are exposed to highpower electromagnetic wave threats because they are controlled by electrical / electronic circuits and wired / wireless communications, along with the development of ICT technology. In particular, most critical communications facilities, such as the Internet Data Center (IDC), are now largely defenseless to high-power electromagnetic pulse attacks.

Recently, protection measures against EMP threats are changing from protection facilities to equipment and technology based protection measures. There is a disadvantage that the installation cost or the operation cost is expensive and the protection facility cannot be moved when the equipment moves. Protection equipment and technology are less expensive than protection facilities, and mobility is easy when the location of the system changes. The paper [16] presented at the UNESST 2018 conference proposed a layered defense scheme that establishes the protection measures from the outside to the system in internal office. The scheme divides defense zones for effective protection, and radiative threats are protected from external protection facilities, and conductive threats are protected by internal protection equipment and technologies. In this paper, we propose a process to determine the most appropriate protection measures for the layered defense scheme. This process determines the scope of the system to be protected, performs a risk assessment for the system, identifies the level of protection, and selects a protection measure that can reduce the risk to the protection level. In addition, it divides the protection measures by the defense zone, performs the cost effectiveness analysis, and decides the appropriate protection measures considering the intention of the commander and the utilization and contribution of the system.

\section{ACKNOWLEDGEMENT}

This paper is a revised and expanded version of a paper entitled [Plan of Protection Measures Construction for Military Information and Communication System against EMP Threats] presented at [UNESST2018, Vietnam and 12 14 July]. This work was supported by the National Research Foundation of Korea Grant funded by the Korean Government (NRF-2016R1D1A1B03934549).

\section{REFERENCES}

[1] Won BC, “Cyber security hot issue EMP attack, how to stop?", Security News, (2017), available online: https://www.boannews.com/media/view.asp?idx=58243, last visit:11.06.2018

[2] You YK, "What measures did the North threaten the 'EMP attack'?", SBS News, (2017), available online: http://news.sbs.co.kr/news/endPage.do?news_id=N1004382879, last visit: 11.06.2018.

[3] Cha JH \& Kim DS, "Concept of nuclear EMP and introduction of protection technology", Defense \& Technology, (2017), 458, 102-109.

[4] Choi HS \& Song TS, "Introduction of Electromagnetic Pulse (EMP) Protection Facility", Building construction, (2016), vol. 16, 3, 22-27. 
[5] Kim HS, “The Actual Situation and Development Plan of EMP Threat”, Defense \& Technology, (2013), 414, 98-103.

[6] Chung YC, "Problems and countermeasures of high-power electromagnetic wave policy", The Journal Of Korean Institute of Electromagnetic Engineering and Science, vol. 28, 3, 3-10.

[7] Kim JJ, "Prevention Methods to defeat Jamming Attacks", International Journal of Security Technology for Smart Device, (2016), vol.3, 2.

[8] Park WC, "EMP Threats and Protective Facilities Test Evaluation", The Magazine of the Institute Of Electronics Engineers Of Korea, (2014), vol. 41, 7, 68-81.

[9] Kwon JH, "R \& D trend of high power non-nuclear electromagnetic wave countermeasure technology", The Journal of Korean Institute of Electromagnetic Engineering and Science, vol. 28, 3, 28-35.

[10] KCA, "Protection technology for the threat prevention of the electronic system of high altitude nuclear electromagnetic pulse (HEMP)", Broadcasting technology and issues, (2013), 22, 1-13.

[11] Min KR, Study on high-power electromagnetic wave protection method for information equipment, KISA Publisher, (2015).

[12] William Radasky \& Edward Savage, Intentional Electromagnetic Interference (IEMI) and Its Impact on the U.S. Power Grid, Metatech Publisher, (2010).

[13] Jin JH, "HEMP filter development trend and design method", The Magazine of the INSTITUTE OF ELECTRONICS ENGINEERS OF KOREA, (2014), vol. 41, 7, 45-58.

[14] Jung SH, Improvement of High Power Electromagnetic Wave Protection Capacity of National Infrastructure Control System, Kyoungi univ. Publisher, (2017).

[15] Kim SU, EMI, EMC, EMP shielding facility construction technology development plan, KAIA publisher, (2017).

[16] Park JC, Cheon SP \& Eom JH, "Plan of Protection Measures Construction for Military Information and Communication System against EMP Threats" The 10th International Conference on u- and e- Service, Science and Technology, Vol.9, No.4, (2018), pp.7-12, http://dx.doi.org/10.21742/ijeic.2018.9.4.02 
International Journal of Advanced Science and Technology Vol.124 (2019) 